Results FEV1 decreased significantly with the cumulative exposure and mean exposure levels. The estimated decrease was close to $200 \mathrm{~mL}$ per year of high exposure, which corresponds roughly to levels of wheat dust higher than $10 \mathrm{mg} / \mathrm{m}^{3}$. Peak expiratory flow and several acute symptoms correlate with recent exposure level. Recovery of the respiratory function six months after exposure to wheat dust and evolution of exposure indicators in workers blood ( $\operatorname{IgG}$ and $\mathrm{IgE}$ ) will be discussed.

Conclusions These results show a chronic effect of exposure to wheat dust on bronchial obstruction. Short term effects and reversibility will be assessed using the full study results.

\section{A NEW, EFFICIENT WEB-BASED TOOL TO COLLECT AND CODE LIFETIME JOB HISTORIES IN LARGE POPULATION- BASED STUDIES: THE COPD PROJECT IN THE UK BIOBANK COHORT}

Sara De Matteis, Lesley Rushton, Debbie Jarvis, Magda Wheatley, Hadia Azhar, Paul Cullinan. Imperial College, London, UK

10.1136/oemed-2014-102362.59

Objectives The manual collection and coding of job histories is the standard method for assessing occupational exposure, but may be infeasible for large population-based studies such as the UK Biobank cohort. We aimed to develop a new web-based tool to automatically collect and code individual lifetime job histories in the UK Biobank cohort for investigating the causes and burden of work-related COPD in the UK.

Method UK Biobank is a population-based cohort of 502682 subjects, aged 40-69 years, recruited in 2006-2010. Baseline spirometry data, current employment and smoking histories were collected. We developed a job questionnaire based on the hierarchical structure of the standard occupational classification (SOC) 2000 to allow participants to automatically self-collect and code their lifetime job histories. The web-based prototype (www.imperial.ac.uk/biobank/questionnaire) was pre-piloted in May-August 2013 among key job sectors using snowball sampling together with a feedback survey.

Results 171 subjects participated in both the pre-piloting and feedback survey. 91\% completed the questionnaire in $<20 \mathrm{~min}$, $85 \%$ considered the instructions clear, and $80 \%$ found their job categories/titles easily. Overall, 96\% judged the questionnaire to be clear and easy. A revised questionnaire has now been designed and will be accessible from different media including PCs/laptops, tablets and smart phones to encourage high response. A demonstration version will be made available to conference participants. Conclusions Our web-based job questionnaire is an efficient new standard tool for collecting and automatically coding lifetime job histories in large population-based studies and is adaptable for use in many occupational health research projects.

\section{USE OF A PREDICTION MODEL OF ASTHMA WITH ANTIASTHMA DRUG CLAIMS FOR EPIDEMIOLOGICAL SURVEILLANCE OF ASTHMA IN SELF-EMPLOYED WORKERS IN FRANCE}

${ }^{1}$ Yuriko Iwatsubo, ${ }^{1}$ Marie Houot, 'Delphine Lauzeille, ${ }^{2}$ Claudine Kamali, ${ }^{1}$ Frédéric Moisan, ${ }^{1}$ Ellen Imbernon. ${ }^{1}$ French Institute for Public Health Surveillance, Saint-Maurice, France; ${ }^{2}$ Régime Social Des Indépendants, La Plaine Saint-Denis, France

10.1136/oemed-2014-102362.60
Objectives To estimate prevalence and risk of current asthma among affiliates to the health insurance for self-employed workers according to economic activities.

Method We defined current asthma using a prediction model developed in a study conducted in 2006 among workers aged between 18 to 65, affiliated to the Régime Social des Indépendants (RSI) in three French regions. The model used as predictors antiasthma drug claims data and the prescriber's medical speciality. In 2013, we obtained from the RSI, economic sectors and drug claims data of all French affiliates on whom we applied our prediction model. We used logistic regression to estimate asthma risk of each economic sector versus all the others.

Results The population comprised 967391 workers. In men, the asthma prevalence was $5.6 \%$. Elevated odds-ratios were observed in the production of food products $(\mathrm{OR}=1.70$ [95\% CI $1.63-$ 1.78]), recycling $(\mathrm{OR}=1.44$ [95\% CI 1.23-1.70]), health and social work $(\mathrm{OR}=1.34$ [95\% CI 1.16-1.54]) and land transport $(\mathrm{OR}=1.08$ [95\% CI 1.03-1.13]). In women, the asthma prevalence was $7 \%$. High odds-ratios were observed in education $(\mathrm{OR}=1.27$ [95\% CI 1.08-1.50]), manufacture of medical and precision instruments $(\mathrm{OR}=1.25 \quad[95 \%$ CI $0.99-1.58])$, land transport $(\mathrm{OR}=1.11$ [95\% CI $0.98-1.25])$ and hotel/restaurants (OR=1.10 [95\% CI 1.05-1.15]).

Conclusions Prevalence estimated by the model was close to that observed among self-employed workers within a national survey conducted in 2003. Elevated risks were observed in several industries known to be at risk but also in those not expected. Prediction model approach will allow asthma surveillance in workers without interview with health insurance organisation data when occupational data are available.

\section{PREDICTORS OF SICKNESS ABSENCE IN PREGNANCY- A DANISH COHORT STUDY}

${ }^{1}$ Mette Lausten Hansen, ${ }^{1}$ Ane Marie Thulstrup, ${ }^{2}$ Jette Kolding Kristensen, ${ }^{3}$ Mette Juhl, ${ }^{4}$ Cecilia Høst Ramlau-Hansen. 'Department of Occupational Medicine, Danish Ramazzini Center, Aarhus University Hospital, Aarhus, Denmark; ' Department of Public Health, Section of General Practice, Aarhus University, Aarhus, Denmark; ${ }^{3}$ The Research Unit Women's and Children's Health, The Juliane Marie Center, Rigshospitalet, Copenhagen, Denmark; ${ }^{4}$ Department of Public Health, Section for Epidemiology, Aarhus University, Aarhus, Denmark

\subsection{6/oemed-2014-102362.61}

Objectives To investigate if parity, fertility treatment, body mass index (BMI), time to pregnancy (TTP), and engagement in physical exercise are risk factors for sickness absence during pregnancy weeks 10 to 30 .

Method We use data from The Danish National Birth Cohort (DNBC) and the Danish Register for Evaluation of Marginalisation (DREAM). DNBC contains information on 100418 pregnancies included from 1996 until 2002. Around pregnancy weeks $12-16$ the participants were interviewed by telephone and provided information on potential predictors, on occupational exposures, lifestyle factors and health. We excluded women, who were no longer pregnant at the time of the interview, were unemployed, multiple pregnant or had an obstetrical event within one year before the DNBC pregnancy. This resulted in a study population of 65047 pregnancies. Outcome data were retrieved from DREAM, which contains information on sickness absence on a weekly basis. Data will be analysed using multivariate logistic regression models. 